\title{
Analysis of Polarization Properties of Armored Target Basing on pBRDF
}

\author{
XU Fei-fei, a ${ }^{1,}$ ZENG Zhao-yang ${ }^{2}$ and CHEN Hang ${ }^{1}$ \\ ${ }^{1}$ Company of Postgraduate Management, the Academy of Equipment, Beijing 101416, China \\ ${ }^{2}$ Department of Photoelectric Equipment, the Academy of Equipment, Beijing 101416, China \\ azyxufei@126.com
}

Keywords: Armored Target, pBRDF Model, Polarization Properties

\begin{abstract}
The polarization properties of the key components of armored target have been researched in this paper. As a new target detection method, the polarization imaging detection can overcome the deficiencies of the traditional detection method in distinguishing the details of armored target. Firstly, make a brief introduction to pBRDF. Furthermore, we collected information such as material, the complex refractive index, roughness of different parts of the armor target, and built the polarization BRDF model. Finally, we obtained its polarization characteristic curve by MATLAB, found that the polarization properties of different parts have obvious difference.
\end{abstract}

\section{Introduction}

As the development of camouflage cover, electromagnetic interference and stealth technology, accurate detection and identification of the armored target is becoming more difficult. Laser polarization imaging detection is a hot topic in recent research because of its advantage on recognizing camouflage target, penetrating the fog and identifying the target details [1]. AFRL (Air Force Research Laboratory) had done the experimental study on the polarization characteristics of standard coating in 2000 and draw a conclusion that paint surface features affect the polarization characteristics [2]. Richard G. Priest and Thomas A. Germer established the microfacet polarization combining with the polarization theory, verified the accuracy of the polarization model by experimental verification [3].In 2005, Shell 11JR combined the BRDF (bidirectional reflectance distribution function) and the theory of stokes, polarization BRDF radiation spectrum control equation is deduced [4].

In this paper, we researched the polarization properties of three materials from the key components of armored target. As the main parameters affecting the polarization state, the complex refractive index and roughness of the three materials will be focused the analysis on.

The rest of the paper is organized in the following sequence. In Section 2, the pBRDF model is introduced in detail. In Section 3, numerical simulation results are made and given to demonstrate the difference of polarization characteristics between the different materials. Concluding remarks are summarized in the final section.

\section{Polarized Bidirectional Reflectance Distribution Function Model}

The polarized bidirectional reflectance distribution function is determined by the surface roughness, refractive index and dielectric constant factors of the target. It can reflect the optical properties of the material surface [5]. We used microfacet theory to build the polarization BRDF model in two aspects, specular reflection and diffuse reflection. The polarization model $F_{r}$ expressed as [6]:

$$
F_{r}=F_{s}+F_{d}
$$

$F_{s}$ is the component value of specular scattering; $F_{d}$ is the component value of diffuse scattering. A single facet of specular scattering is shown in Fig. 1 


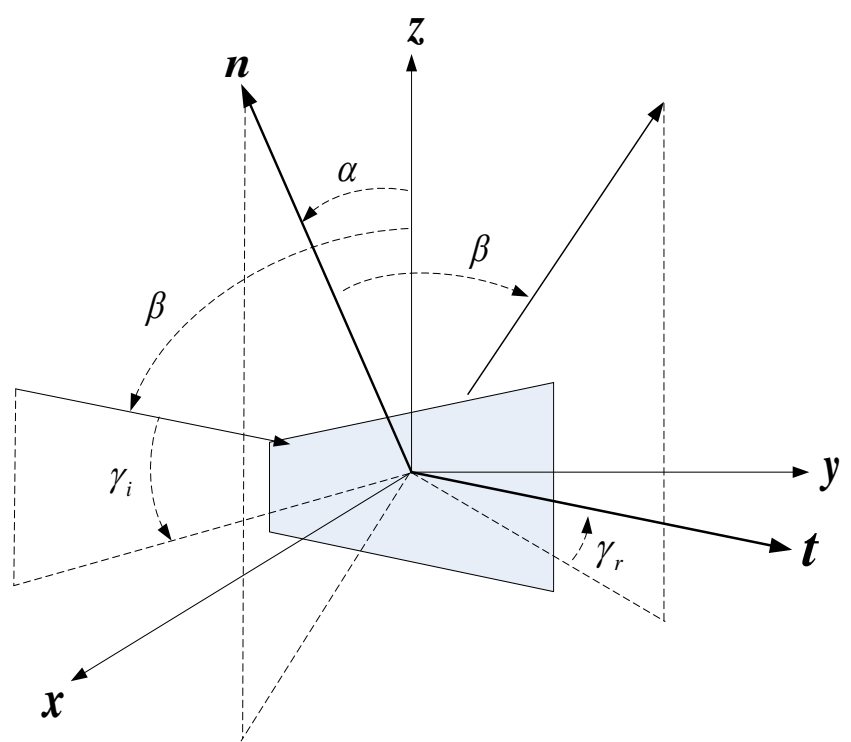

Fig. 1 Geometrical diagram of pBRDF

$n$ is the normal to microfacet, $Z$ is the mean surface normal, $\alpha$ is the angle between $n$ and $Z, \beta$ is the angle between $Z$ and the reflected light.

The specular scattering component $F_{s}$ is calculated by the formula below:

$$
F_{s}=\frac{R_{F}(\beta) P(\alpha) G}{4 \cos \theta_{i} \cos \theta_{r} \cos \alpha}
$$

$R_{F}(\beta)$ is the Fresnel function for the microfacet and related to the complex refractive index of the material; $p(\alpha)$ for surface distribution function, it can reflect the roughness of the surface and be expressed by $\alpha$ and $\sigma$; $G$ for the shielding factor, it can be expressed in the parameters of the $\theta_{\mathrm{i}}, \theta_{\mathrm{r}}$, $\alpha$ and $\beta ; \theta_{\mathrm{i}}$ for the incident angles; $\theta_{\mathrm{r}}$ for the reflex angle; $\sigma$ for the roughness.

The diffuse scattering component $F_{d}$ is calculated by the formula below:

$$
F_{\mathrm{d}}\left(\theta_{i} ; \sigma\right)=\frac{1}{\pi}\left(1-\rho_{D H R}\right) R_{F, 00}
$$

$\rho_{D H R}$ for diffuse scattering component calculated by directional hemispherical reflectance.

As a consequence, according to the formula one, we can get the BRDF energy of the target.

$$
F_{r, 00}=F_{s, 00}+\frac{1}{\pi}\left[1-\rho_{D H R}\right] R_{F, 00} \quad F_{r, j k}=F_{s, j k} \quad j, k \neq 0
$$

$F_{r, 00}$ for the total BRDF energy of the target. Due to the result of the analysis above, the total BRDF energy is related to such parameters as roughness, complex refractive index, incident angle and the scattering angle.

\section{Simulation and analysis}

Through the analysis of domestic and foreign typical armored target structure, determine the key components are armor, turret, barrel tracks, etc. Different parts of the armored vehicles use different materials, cannon gun tube are mainly composed of elements of nickel chromium alloy steel; traditional body armor consists of homogeneous armor steel, but the new generation of armour using combined materials, such as the British Joe Bam armor using ceramic composite materials; Turret and panzer body now using composite materials to reduce weight and improve defensive 
performance, such as Britain and the United States developed together the E-glass fiber reinforced epoxy resin turret[7].

In view of the materials from different parts of the armored vehicle, gathering the materials complex refractive index and the roughness [8], see Table 1 .

Table1 The complex refractive index and roughness of armor materials

\begin{tabular}{ccc}
\hline Armor Material & Complex Refractive Index & $\begin{array}{c}\text { Roughness } \\
\operatorname{Ra}[\mu \mathrm{m}]\end{array}$ \\
\hline alloy steel & $1.24-2.5 \mathrm{i}$ & 0.15 \\
SIC ceramic & 2.635 & 0.03 \\
glass fiber reinforced epoxy resin & 1.55 & 0.05 \\
\hline
\end{tabular}

According to the study of pBRDF model and material information above, using MATLAB software to experiment, analyzing the different polarization characteristics of armored materials. The results are as follows:

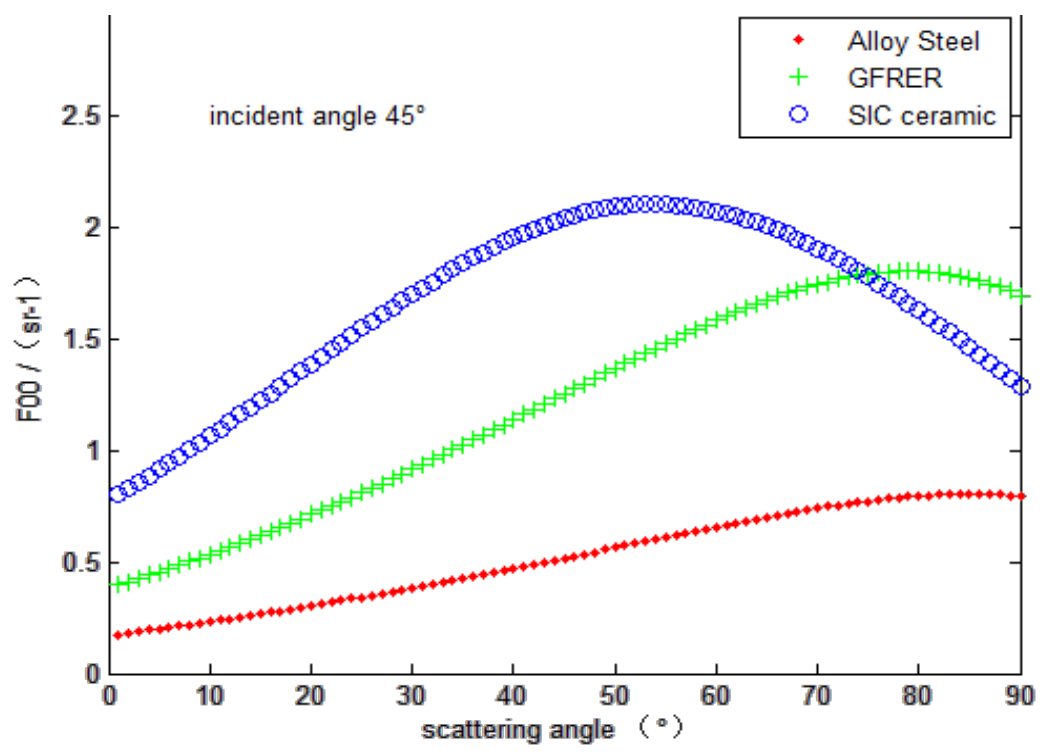

a) $F_{\mathrm{r}, 00}$ for the three materials

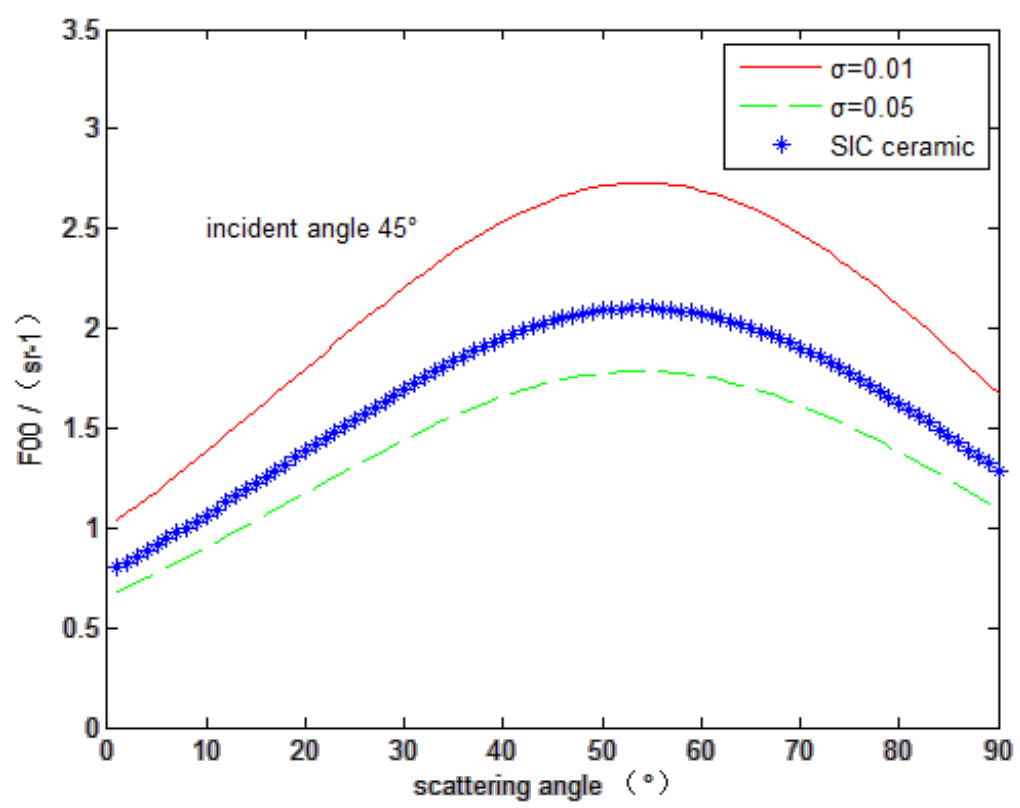

b) $F_{\mathrm{r}, 00}$ for the same material, different roughness 


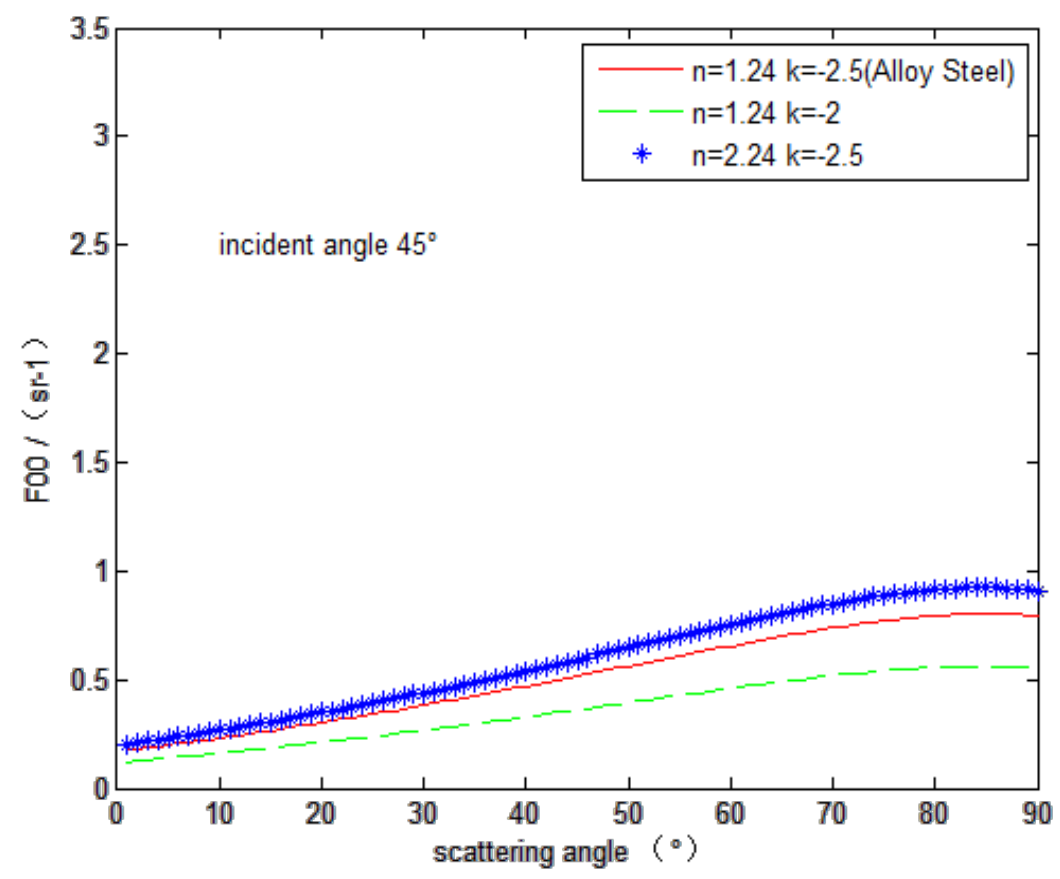

c) $F_{r, 00}$ for the same material, different complex refractive index

Fig.2 Simulation Results

As we can see from Fig.2, the research of polarization energy changing with scattering angle was made on observation angle of 45 degrees. These three different materials have significant differences in polarization energy, $F_{\mathrm{r}, 00}$ increases with the increase of scattering angle until reaching a peak point. SIC ceramic reach the peak point earlier and has the largest peak point. $F_{r, 00}$ for alloy steel is small and the curve is flat.

In order to study the influence of roughness for polarization energy, we endow the different roughness on the SIC ceramic (it is not real, only be used to facilitate the research) [9]. It can be seen that the polarization energy decrease with the increase of roughness, due to the roughness increases, the diffuse scattering increase on the surface of the material, so that reduce the intensity of polarization. Similarly, we endow the different complex refractive index on the alloy steel. It can be seen that when the real component of complex refractive index increases, the polarization energy decreases slightly, but, with the increases of the imaginary part, polarization energy increases obviously.

\section{Summary}

We studied the polarization characteristics of three typical armored materials basing on the pBRDF model. Respectively analyzed the influence of roughness and complex refractive index on the polarization energy, the following conclusions were drawn.

1) The surface roughness and the complex refractive index have an effect on the polarization characteristics of armored target. These factors are decided by the material itself. And therefore, we can distinguish camouflaged target and the real target using their polarization characteristics.

2) The significant differences of polarization characteristics of the key components of armored vehicles can be used in target recognition. Specific components of the armored target will be able to determine, and it will enhance the accuracy of target recognition.

\section{References}

[1] Zhao Y Q, Zhang H C, Pan Q. Based on the polarization characteristics of target detection [J]. Chinese Journal of Quantum Electronics, 2003, 20(6):685-688. 
[2] Goldstein D H. Polarimetric characterization of federal standard paints [C]//International Symposium on Optical Science and Technology. International Society for Optics and Photonics, 2000: 112-123.

[3] Priest R G, Gerner T A. Polarimetric BRDF in the microfacet model: theory and measurements [R]. WASHINGTON DC: NAVAL RESEARCH LAB, 2000.

[4] Shell J R, Schott J R. A polarized clutter measurement technique based on the governing equation for polarimetric remote sensing in the visible to near infrared [C]. Defense and Security. International Society for Optical and Photonics, 2005: 34-45.

[5] H M, JD S, MJ. H. A geometrical optics polarimetric bidirectional reflectance distribution function for dielectric and metallic surfaces.[J]. Optics Express, 2009, 17(24):22138-22153.

[6] G. Gartley M, D. Brown S, D. Goodenough A, et al. Polarimetric scene modeling in the thermal infrared[J]. Proceedings of SPIE - The International Society for Optical Engineering, 2007.

[7] Su J H, Li A P, Ge W Y. The application of composite materials in the tank armored vehicles [J]. Science and technology of China, 2011, (21):378-378.

[8] V T, DG V, CD. C. Polarization-based index of refraction and reflection angle estimation for remote sensing applications.[J]. Appl Opt, 2007, 46(30):7527-7536.

[9] ChenW L, Wang X, Jin Q W. Based on the pBRDF analysis of the infrared polarization characteristics [C]. /The Chinese society of optical optical assembly. 2010. 